\title{
Establishment of a pediatric COVID-19 biorepository: unique considerations and opportunities for studying the impact of the COVID-19 pandemic on children
}

Rosiane Lima ${ }^{1,2+}$, Elizabeth F. Gootkind ${ }^{3 \dagger}$, Denis De la Flor ${ }^{1}$, Laura J. Yockey ${ }^{4,5}$, Evan A. Bordt ${ }^{6}$, Paolo D'Avino ${ }^{1}$, Shen Ning ${ }^{7}$, Katerina Heath ${ }^{8}$, Katherine Harding ${ }^{8}$, Jaclyn Zois ${ }^{1}$, Grace Park ${ }^{3}$, Margot Hardcastle ${ }^{3}$, Kathleen A. Grinke ${ }^{9}$, Sheila Grimmel ${ }^{9}$, Susan P. Davidson ${ }^{9}$, Pamela J. Forde ${ }^{9}$, Kathryn E. Hall ${ }^{9}$, Anne M. Neilan ${ }^{4,10}$, Juan D. Matute ${ }^{11,12}$, Paul H. Lerou ${ }^{11,12}$, Alessio Fasano ${ }^{1,2,12}$, Jessica E. Shui ${ }^{10,12}$, Andrea G. Edlow ${ }^{5,12}$ and Lael M. Yonker ${ }^{1,3,12^{*}}$ (D)

\begin{abstract}
Background: COVID-19, the disease caused by the highly infectious and transmissible coronavirus SARS-CoV-2, has quickly become a morbid global pandemic. Although the impact of SARS-CoV-2 infection in children is less clinically apparent, collecting high-quality biospecimens from infants, children, and adolescents in a standardized manner during the COVID-19 pandemic is essential to establish a biologic understanding of the disease in the pediatric population. This biorepository enables pediatric centers world-wide to collect samples uniformly to drive forward our understanding of COVID-19 by addressing specific pediatric and neonatal COVID-19-related questions.
\end{abstract}

Methods: A COVID-19 biospecimen collection study was implemented with strategic enrollment guidelines to include patients seen in urgent care clinics and hospital settings, neonates born to SARS-CoV-2 infected mothers, and asymptomatic children. The methodology described here, details the importance of establishing collaborations between the clinical and research teams to harmonize protocols for patient recruitment and sample collection, processing and storage. It also details modifications required for biobanking during a surge of the COVID-19 pandemic.

Results: Considerations and challenges facing enrollment of neonatal and pediatric cohorts are described. A roadmap is laid out for successful collection, processing, storage and database management of multiple pediatric samples such as blood, nasopharyngeal and oropharyngeal swabs, sputum, saliva, tracheal aspirates, stool, and urine. Using this methodology, we enrolled 327 participants, who provided a total of 972 biospecimens.

(Continued on next page)

\footnotetext{
* Correspondence: lyonker@mgh.harvard.edu

${ }^{+}$Rosiane Lima and Elizabeth F. Gootkind co-first authors

'Mucosal Immunology and Biology Research Center, Massachusetts General

Hospital, Boston, MA, USA

${ }^{3}$ Department of Pediatrics, Pulmonary Division, Massachusetts General Hospital, Boston, MA, USA

Full list of author information is available at the end of the article
}

(C) The Author(s). 2020 Open Access This article is licensed under a Creative Commons Attribution 4.0 International License, which permits use, sharing, adaptation, distribution and reproduction in any medium or format, as long as you give appropriate credit to the original author(s) and the source, provide a link to the Creative Commons licence, and indicate if changes were made. The images or other third party material in this article are included in the article's Creative Commons. licence, unless indicated otherwise in a credit line to the material. If material is not included in the article's Creative Commons licence and your intended use is not permitted by statutory regulation or exceeds the permitted use, you will need to obtain permission directly from the copyright holder. To view a copy of this licence, visit http://creativecommons.org/licenses/by/4.0/ The Creative Commons Public Domain Dedication waiver (http://creativecommons.org/publicdomain/zero/1.0/) applies to the data made available in this article, unless otherwise stated in a credit line to the data. 


\begin{abstract}
(Continued from previous page)
Conclusions: Pediatric biospecimens will be key in answering questions relating to viral transmission by children, differences between pediatric and adult viral susceptibility and immune responses, the impact of maternal SARS-CoV-2 infection on fetal development, and factors driving the Multisystem Inflammatory Syndrome in Children. The specimens in this biorepository will allow necessary comparative studies between children and adults, help determine the accuracy of current pediatric viral testing techniques, in addition to, understanding neonatal exposure to SARSCoV-2 infection and disease abnormalities. The successful establishment of a pediatric biorepository is critical to provide insight into disease pathogenesis, and subsequently, develop future treatment and vaccination strategies.
\end{abstract}

Keywords: COVID-19, SARS-CoV-2, Multisystem inflammatory syndrome in children (MIS-C), Viral transmission, Viral susceptibility, Biorepository, Biobank, pediatric

\section{Background}

The global pandemic of COVID-19, caused by the highly infectious and transmissible coronavirus, SARS-CoV-2, has become a leading cause of death in older adults [1]. While adults can develop life-threatening complications such as pneumonia, acute respiratory distress syndrome (ARDS), and sepsis from SARS-CoV-2 infection, its impact on children is less clinically apparent and needs to be studied. Collecting high-quality biospecimens from infants, children and adolescents in a standardized manner during the COVID-19 pandemic is essential for understanding the biologic consequences of SARS-CoV-2 infection in children.

Specific questions that must be addressed revolve around the role children play in viral transmission, differences in pediatric viral susceptibility and immune responses, which could guide potential therapies for adults, the impact of maternal SARS-CoV-2 infection on fetal development, and factors driving the development of severe hyperinflammatory shock and cardiac damage seen in Multisystem Inflammatory Syndrome in Children (MIS-C). Outlined here is a roadmap for establishing a biorepository of specimens obtained from infants, children and adolescents during the COVID-19 pandemic. Special attention is provided to pediatric-specific considerations in the establishment of a biorepository during the COVID-19 pandemic. The goal is to enable pediatric centers world-wide to collect samples in a standardized manner to drive forward our understanding of COVID-19.

\section{Methods}

The impact of SARS-CoV-2 infection on infants and children is not well-defined. Children are typically asymptomatic or mildly symptomatic during the acute infection, although some can develop significant complications requiring intensive care. In order to capture the full range of SARS-CoV-2 infection in the pediatric population, a COVID-19 biospecimen collection study was designed and implemented, including patients seen in urgent care clinics and hospital settings, neonates born to SARS-CoV2-infected mothers, and asymptomatic children. Each study population required specific tailoring of study conduct to effectively and efficiently collect critical samples.

Cornerstones of the biorepository included open dialogue between research and clinical team members, a sensitivity to procedures required for specimen collection in children, and clear documentation of study participation and sample collection. Close communication and collaborations with the adult COVID-19 biorepository enable paralleled recruitment efforts and processing procedures and ensured consistency and harmonization across patient cohorts, facilitating high-quality comparisons between patient groups and with adult cohorts. Central to the operation, physician-scientists in Pediatrics, Neonatology, Medicine-Pediatrics, and Obstetrics-Gynecology harmonized sample collection protocols, established clinical connections and provided clinical and scientific context to COVID-19-related research in the neonatal and pediatric population.

\section{Ethical and biosafety review board processes}

To establish a Pediatric COVID-19 biorepository during the surge of COVID-19 cases locally, protocols were rapidly submitted to our Institutional Biosafety Committee (IBC, MGH IBC\#2020B000061) and the Institutional Review Board (IRB, MGH IRB\#2020P000955) for approval. During this initial wave of the COVID-19 pandemic in late March 2020, COVID-19 research proposals were prioritized by the IBC and IRB. A biosafety protocol was submitted to the IBC to transition from a Biosafety Level 2 (BSL2) to an enhanced BSL2+ laboratory environment, allowing collection, processing, and storage of SARS$\mathrm{CoV}$-2-infected samples. IBC approval was obtained within 2 weeks. An expedited IRB review facilitated IRB approval 2 days following submission. Figure 1 displays a timeline of study activity relative to the community surge of COVID-19 cases in Massachusetts.

\section{Patient enrollment and sample collection}

Eligible participants in all cohorts were identified by screening outpatient clinic schedules or hospital admission lists, then discussing potential patients with care 


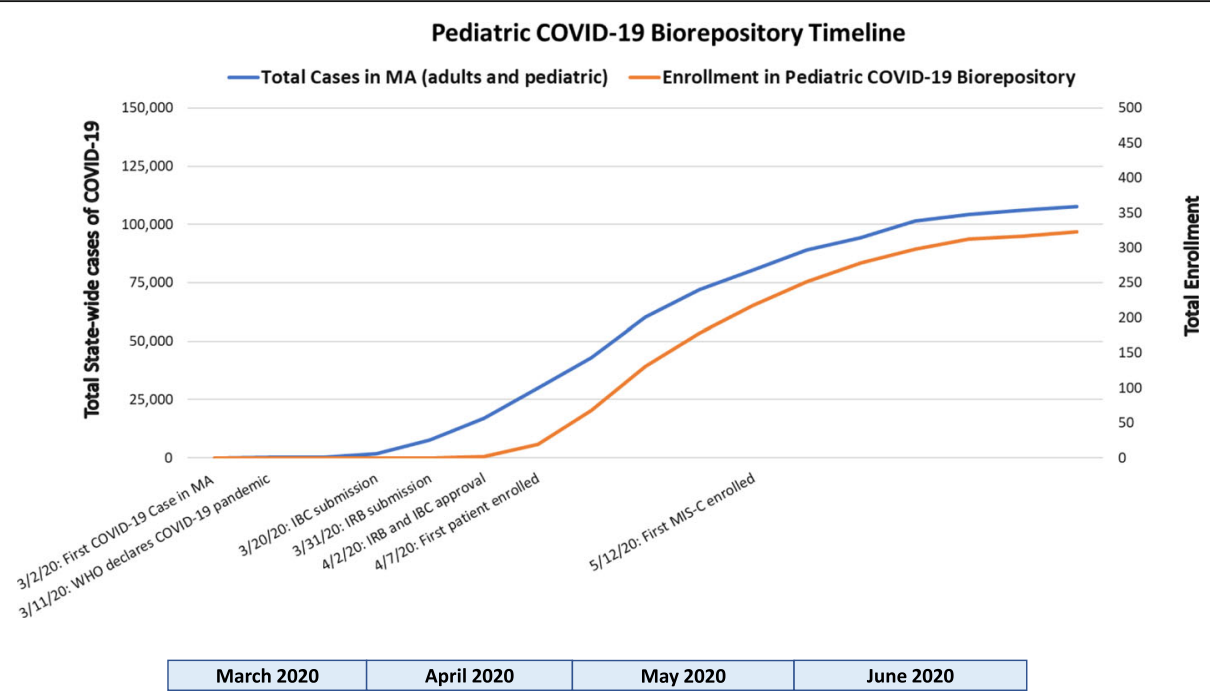

Fig. 1 Pediatric COVID-19 Biorepository timeline of study implementation and enrollment relative to the community surge of COVID-19 cases in the state of Massachusetts [2]

team members. If appropriate, parents/guardians or patients (if $\geq 18$ years of age) were called by phone to introduce the study and, if interested in participation, an informed consent was completed by phone. Participants or their parent/guardian also selected which biospecimens they would provide to the biorepository. Assent was completed by phone, when possible, with parents/ guardian present for children 7-17 years of age. Inperson consent/assent was waived by the IRB to avoid close contact between patients and research staff and to abide by the social distancing measures implemented by the state. Witness requirements were also waived as a result of the restricted visitor hospital policy due to the pandemic. One copy of the signed consent form, and assent form if appropriate, was emailed to enrolled patients or parents/guardians, and another was uploaded into the electronic medical record flagging the patient as a research participant, clearly documenting research participation for the clinical care teams facilitating sample collection. Paper copies were not provided to participants due to the COVID-19 restrictions. Upon enrollment, participants were assigned a unique study ID number using REDCap, a secure, centralized online database platform that allows simultaneous recruitment at multiple sites without risking assigning the same number to multiple patients.

In order to include pediatric and neonatal patients from a range of clinical presentations for COVID-19, we established 4 cohorts of patients from ages 0-25 years, reflecting the ages of patients cared for by the pediatric teams during the surge of COVID-19 cases locally: 1) Pediatric patients with mild-moderate COVID-19, presenting to the MGH COVID-19 urgent care clinics, 2) Pediatric patients with severe COVID-19 or MIS-C requiring hospitalization, 3) Newborns born to mothers infected with SARS-CoV-2 at any point during their pregnancy and infants born to non-infected mothers, and 4) asymptomatic children presenting to their wellvisits during the pandemic.

Each cohort presented unique challenges and required tailored strategies for a successful recruitment. In the pediatric urgent care units, challenges in enrollment included variability in patient volume and frequent rotation of nurses, medical assistants, and physicians. Research coordinators had to be flexible to adapt to the frequently changing workflow within the clinic. For enrollment in the hospitalized cohort, the research team remained attentive to new admission lists and promptly completed enrollment protocols in order to obtain specimens prior to the initiation of treatment, as interventions such as intravenous immunoglobulin or steroids would interfere with the natural immune responses to SARS-CoV-2 infection. Specimen collection from the hospitalized cohort was coordinated with clinical laboratory collections to minimize blood draws and collection procedures. For enrollment of newborns, the research coordinators coordinated with maternal arm of the adult COVID-19 biorepository to allow simultaneous enrollment of mother and newborns prior to birth facilitating the recruitment process and limiting non-clinical interactions with the mother during the perinatal period. Additionally, blood volumes collected for newborns were minimized to $<1 \mathrm{ml}$ obtained by heal stick and coordinated with blood collection for newborn screening. Enrolling well-visits was challenging as blood draws are not routinely obtained in all ages and children are often unwilling to undergo voluntary venipuncture. In each cohort, the research staff was mindful of the physical and 
emotional stress the care teams were enduring while caring for patients with COVID-19 and therefore sought to minimize disruptions in clinical care. Figure 2 provides a schematic of the recruitment strategy.

\section{Pediatric patients with mild-moderate COVID-19}

As most children did not require hospital-level care, significant efforts were made to enroll patients in the outpatient setting. COVID-19 screening clinics, called Respiratory Infection Control clinics, were established at Massachusetts General Hospital. As COVID-19 symptoms are non-specific and current diagnostic reporting is time-delayed, all patients presenting to the pediatric COVID-19 screening clinics were eligible to participate in the biorepository. During the surge of COVID-19 cases locally, young adults up through 25 years of age were seen in the pediatric COVID-19 clinics.

For patients seen in the Respiratory Infection Control clinics, the CRC called eligible participants via telephone after acquiring approval from the lead physicians in the clinics. After acquiring verbal consent over the telephone, the CRC alerted the clinical team of patient enrollment and the clinical teams obtained specimens for research. Participants who provided informed consent could give nasopharyngeal, oropharyngeal swabs, and/or blood. Stool and urine were not collected given time limitations of clinic visits and patient flow patterns established to minimize potential COVID-19 exposures to clinical staff. Blood was collected into one tube with an EDTA anticoagulant (EDTA tube) (BD), one serum separator tube (SST) (BD), and a PAXgene RNA tube (BD). Blood volumes varied, depending on the age and weight of the patient, in accordance with limits established by the IRB. The aerosolizing procedure of collecting nasopharyngeal and oropharyngeal swabs into $15 \mathrm{~mL}$ falcon tubes, containing $3 \mathrm{~mL}$ phosphate buffered saline (PBS) (Gibco), was performed by clinical team members wearing N95 mask, face shield, protective outer gown, and disposable gloves.

Patients with severe COVID-19 or multisystem inflammatory syndrome in children requiring hospitalization

Pediatric patients who were hospitalized with suspicion of SARS-CoV-2 exposure and/or symptoms concerning for SARS-CoV-2 infection or MIS-C were identified by members of the research team, who subsequently requested approval from the clinical team to approach the patient. A member of the research team contacted the patient and family via phone to obtain informed consent, as described above, and coordinated with both the clinical and TCRC teams for specimen collection. Sample collection was pre-planned with the clinical teams via emails and occurred every $2-3$ days for this cohort of patients. Hospitalized patients could opt to provide urine, stool, sputum, or if intubated, tracheal aspirates, in addition to blood, nasopharyngeal and oropharyngeal swabs. Phlebotomy was aligned with clinical blood draws, when feasible, although participants had an option to undergo a separate venipuncture for research purposes. Blood was collected into an EDTA tube, an SST tube, and a PAXgene RNA tube. Repeat samples were collected on alternating days, as feasible. Based on daily coordination between the research and clinical teams, discarded blood from clinical labs were also obtained from hospitalized patients.

\section{Newborns born to mothers with and without SARS-CoV-2 infection}

Pregnant women with confirmed SARS-CoV-2 infection followed in the MGH obstetrics practice, presenting to the Labor and Delivery (L\&D) Unit, or hospitalized for SARS-CoV-2 illness, were approached to enroll their

\begin{tabular}{|c|c|}
\hline \multicolumn{2}{|c|}{ Pediatric Biorepository Enrollment } \\
\hline Hospitalized Children & Neonates \\
\hline $\begin{array}{l}\text { Description: } \\
\text {-All pediatric SARS-CoV-2 (+) patients } \\
\text {-Children under investigation for COVID-19 } \\
\text { Multisystem Inflammatory Syndrome in Children } \\
\text { Considerations and Challenges: } \\
\text {-Coordinate biospecimen collection with clinical care } \\
\text {-Collect biospecimens prior to treatment initiation }\end{array}$ & $\begin{array}{l}\text { Description: } \\
\text {-Babies born to SARS-CoV- } 2(+) \text { and SARS-CoV-2 (-) } \\
\text { mothers during COVID-19 crisis } \\
\text { Considerations and Challenges: } \\
\text {-Simultaneous enrollment of mother } \\
\text { and child prior to birth } \\
\text {-Low blood volumes, time-sensitivity }\end{array}$ \\
\hline Pediatric Urgent Care & Pediatric Well-Visits \\
\hline $\begin{array}{l}\text { Description: } \\
\text {-Pediatric patients with viral symptoms, } \\
\text { concern for SARS-CoV-2 infection } \\
\text { Considerations and Challenges: } \\
\text {-Coordinate biospecimen collection with clinical care } \\
\text {-Variable patient volume, frequent staffing changes }\end{array}$ & $\begin{array}{l}\text { Description: } \\
\text { •Healthy children at well-visits during COVID-19 crisi } \\
\text { Considerations and Challenges: } \\
\text {-Collect potential exposure history } \\
\text {-Labs not always collected at well-visits, } \\
\text { difficult to consent for research-only draw }\end{array}$ \\
\hline
\end{tabular}

Fig. 2 Schematic of the recruitment strategies used to pursue the collection of pediatric samples for the Pediatric COVID-19 biorepository (Created with BioRender.com) 
infant in the Pediatric COVID-19 Biorepository following birth in collaboration with the maternal arm of the Adult COVID-19 biorepository. When universal screening for SARS-CoV-2 infection was initiated on all pregnant women admitted to $L \& D$, asymptomatic SARS-CoV-2 positive patients were identified and offered enrollment. Women who tested negative for SARS-CoV-2 were also approached as a control group. The pregnant mothers were simultaneously offered enrollment in the companion Obstetric COVID19 Biorepository, which included collection of placental biopsies, umbilical cord blood, and other maternal samples. The clinical team assessed the patient's interest in the Biorepository, then a member of the research staff contacted the patient via telephone to obtain informed consent. Parents could opt to have newborn blood, nasopharyngeal and oropharyngeal swabs, urine, stool, and (if intubated) tracheal aspirates collected. All samples were collected in the clinical setting by the clinical team members to accommodate COVID-19 infection control guidelines, minimizing the risk of SARS-CoV-2 transmission and limit personal protective equipment (PPE) use.

Blood was collected via heel stick between 24 and $36 \mathrm{~h}$ of life, simultaneously with the heel stick for clinical newborn screening, into two EDTA microtainer tubes (BD). Research nasopharyngeal and/or oropharyngeal swabs were obtained after $24 \mathrm{~h}$ of life, batched at the time of the nasopharyngeal swab for SARS-CoV-2 testing, if performed clinically. Stool and urine were collected on day of life 0 and 2. Stool was collected directly from the diaper. Urine was collected by placing cotton balls in the diaper, then transferring the urine-soaked cotton balls into a specimen cup for transport. If the recruited newborn was intubated for clinical indications, tracheal aspirates were collected at the time of clinical suctioning.

\section{Asymptomatic children presenting to their well-visit during the COVID-19 pandemic}

Children presenting for their 2-, 3-, or 4-year annual well-child visit with their pediatrician for planned phlebotomy were eligible to participate in this cohort. Eligible patients were identified by study staff and clinicians. If appropriate, researchers contacted the parents via telephone prior to their visit to explain the research and obtain informed consent. Blood and saliva were collected during their clinical phlebotomy. Saliva collection is not considered an aerosolizing procedure; thus, these specimens could be collected in clinic without the need for N95 mask use. The specimens were immediately transported to the laboratory for processing.

\section{Data collection}

REDCap databases were used to record all study data, including: 1) An enrollment log serving as the decoding $\log$ - study ID numbers were assigned consecutively across all four patient groups; 2) A laboratory processing database with pertinent processing and freezer storage location information; 3) A chart review database, with demographic and clinical data, including COVID-19 exposures, SARS-CoV-2 polymerase chain reaction (PCR) results, symptoms, and outcomes; 4) A questionresponse database about COVID-19 exposures and risk factors, specifically for the well-visit cohort.

\section{Specimen transport and processing}

In accordance with specimen transport guidelines, specimens were sealed in a leak-proof container labeled with subject's study ID, then placed in a tight-sealed, biohazard-labeled, secondary container with a rigid outer container and lockable lid (e.g. Igloo cooler) for transport to the laboratory. The entire research team was properly trained on BSL2+ procedures, as required for handling SARS-CoV-2 specimens. A coordinated effort by research personnel enabled successful and efficient troubleshooting, and processing of high influx of samples to the lab during the acute rise of COVID-19 cases in the months of April-June 2020 (Fig. 1). Scheduled shifts were implemented throughout the week to ensure the safety of all research staff and sample processing efficiency. Three laboratory roles were created: 1) blood processing technician with extensive technical skill required for blood cell isolation, 2) biospecimen processing technician fully trained in BL2+ enhancement protocols, and 3) specimen labeling, quality control, and sample storage staff. These roles optimized processing workflow, safety precautions, and resources (including staff resource). Paramount to the success of this biorepository included open communication via emails and the use of mobile group messaging outlets, frequent quality checks between staff regarding data and sample collection and processing, accessible leadership, and coordination with patients' clinical care teams.

Blood samples were processed following BSL2 safety guidelines, with a lab coat, nitrile/latex gloves, and a face shield or safety goggles. All other samples, including nasopharyngeal and oropharyngeal swabs, sputum, saliva, tracheal aspirates, stool, and urine were processed following BSL2+ safety guidelines. BSL2+ safety precautions require all samples to be processed in a certified biosafety cabinet (BSC), class II A2, with intake airflow. Well-trained laboratory personnel handling infectious specimens were required to wear closed-front water impermeable gowns, double nitrile/latex gloves, sleeve covers, and a face shield. Outer gloves were removed 
when moving away from the BSC and replaced with a new glove when returning to work in the BSC.

\section{Plasma}

Blood samples collected in tubes with an EDTA anticoagulant were stored at room temperature until processed, within $24 \mathrm{~h}$ of collection. Tubes were spun at $1000 \mathrm{~g}$ for $10 \mathrm{~min}$ with brake activated. Plasma was then collected, aliquoted, stored at $-80^{\circ} \mathrm{C}$, and logged in the REDCap database (Fig. 3b).

\section{Peripheral blood mononuclear cells (PBMCs)}

Immediately following the removal of plasma, samples with greater than $2 \mathrm{~mL}$ initial volume were processed for PBMC isolation using a Ficoll density gradient [3]. Briefly, blood was transferred into a $50 \mathrm{~mL}$ conical tube, then diluted 1:1 with Hanks' Balanced Salt Solution without calcium or magnesium (HBSS minus) (Gibco). This diluted blood was then gently layered on top of Ficoll-Paque Plus (GE Healthcare) at 2:1 ratio (2 volumes of blood diluted with HBSS minus to 1 volume Ficoll). Careful attention was made to avoid any mixing of blood with the Ficoll layer. The conical tube was then centrifuged at $1000 \mathrm{~g}$ for $30 \mathrm{~min}$ at room temperature with brake inactivated to allow adequate layering of cellular components. The cloudy ring below the plasma and above the Ficoll (i.e. the PBMC layer) was collected and transferred to a new 15 $\mathrm{mL}$ conical tube, with HBSS minus added to bring the volume to $15 \mathrm{~mL}$ (Fig. 3a). This tube was then centrifuged at $330 \mathrm{~g}$ for $10 \mathrm{~min}$, with high brake activated. The supernatant was removed, the PBMC pellet was again washed with HBSS minus, and then resuspended in $10 \mathrm{~mL}$ HBSS minus for counting. Cell count was obtained by diluting $10 \mu \mathrm{L}$ of sample with $90 \mu \mathrm{L}$ of trypan blue, mixed, and sampled on a hemocytometer. Cells were then frozen in freshly-prepared freezing medium (RPMI 1640 Medium with $1 \%$ penicillin-streptomycin, L-glutamine, $1 \%$ sodium pyruvate, $1 \%$ non-essential amino-acids, and 20\% Fetal Bovine Serum (FBS) (Sigma)) with 10\% DMSO (Sigma) for a goal concentration range of 5-10 million cells/vial, placed in a chilled Mr. Frosty filled with isopropanol, then immediately placed at $-80^{\circ} \mathrm{C}$. Final concentration (5-10 million cells per $1 \mathrm{~mL}$ of freezing medium) and number of aliquot vials were logged. The following day, PBMC cryovials were moved to a liquid nitrogen freezer for long term storage, and location was recorded in specimen log.

PBMCs were isolated within $24 \mathrm{~h}$ of phlebotomy, although higher cell counts were obtained if isolated within $3-4 \mathrm{~h}$ of collection. If less than $5 \mathrm{~mL}$ blood was collected, a $15 \mathrm{~mL}$ conical tube, rather than a $50 \mathrm{~mL}$ conical tube could be used for Ficoll layering. Fresh freezing media were made throughout the day for each sample batch.

\section{Neutrophils}

Neutrophils were extracted from the red blood cell layer that remained following the collection of PBMCs (Fig. 3a). Neutrophils were isolated using EasySep Direct Human Neutrophil Isolation Kit (StemCell Technologies). The remaining blood layer was incubated with EasySep Direct RapidSpheres and EasySep Direct Human Neutrophil Isolation Cocktail,

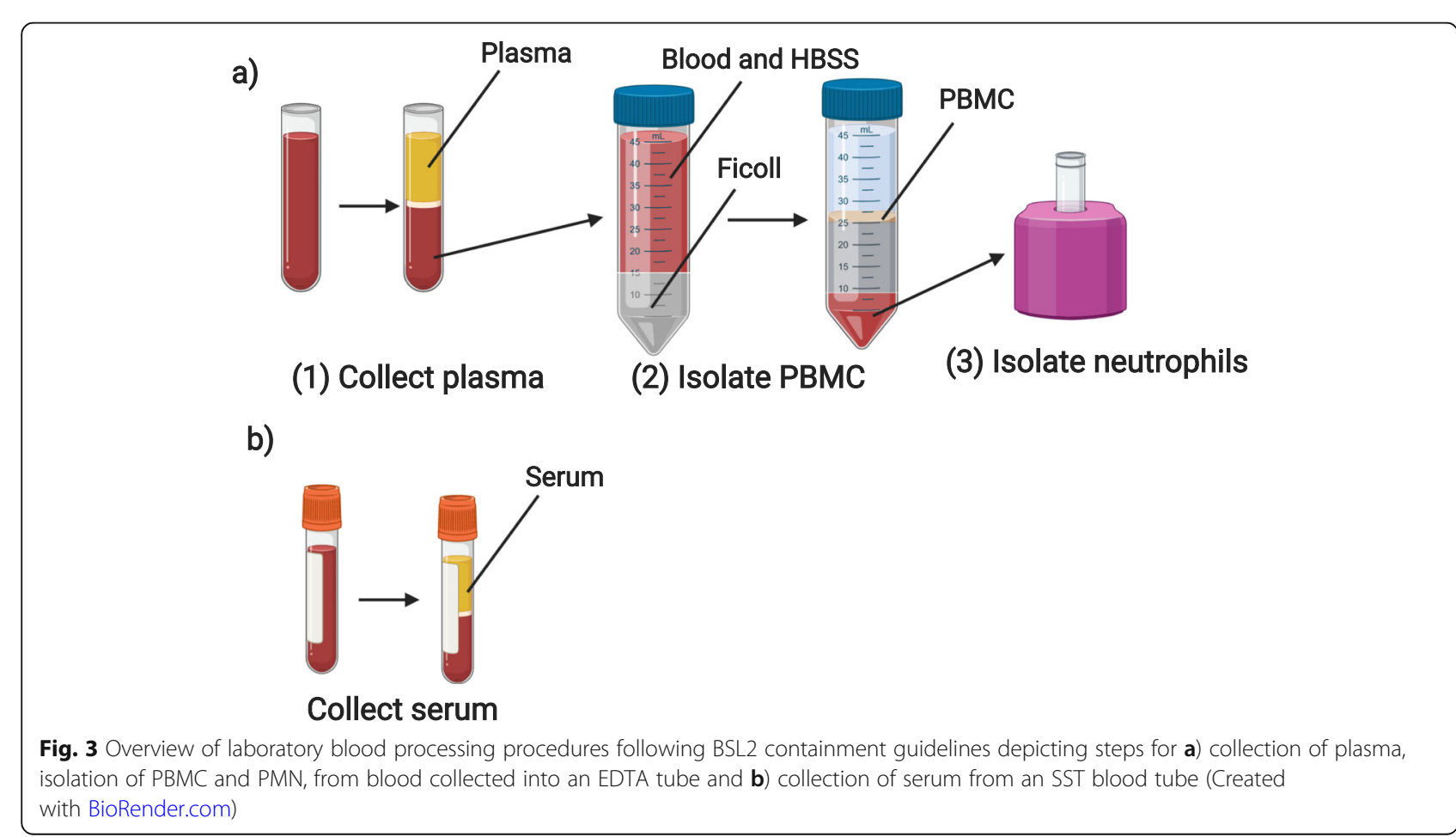


then diluted in EasySep Buffer. Neutrophils were isolated by successive negative magnet selection using EasySep magnets, then counted using a hemocytometer and aliquoted into Eppendorf tubes for RNA extraction $\left(1 \times 10^{5}\right.$ cells/tube) or DNA analysis $\left(5 \times 10^{6}\right.$ cells/tube). Neutrophils designated for RNA extraction were resuspended in $100 \mu \mathrm{L}$ of RNA lysis buffer (TCL) (Qiagen) with 1\% $\beta$-mercaptoethanol (Sigma), immediately stored at $-80^{\circ} \mathrm{C}$ and logged. Neutrophils planned for DNA analysis were pelleted then directly stored at $-80^{\circ} \mathrm{C}$ and logged.

For RNA extraction steps, a cleaning agent, such as RNaseZAP should be used to remove RNAse from the working surface. RNA lysis buffer should be newly made for each sample using a 10:1 TCL to $\beta$-mercaptoethanol ratio.

\section{Serum}

Serum samples were collected from blood drawn into serum separator tubes without any anticoagulant (BD).
Blood was kept at room temperature, standing upright for $30-60 \mathrm{~min}$, then spun at $1200 \mathrm{~g}$ for $10 \mathrm{~min}$ with brake activated. Serum was then collected, aliquoted, stored, and logged (Fig. 3b).

\section{Nasopharyngeal and Oropharyngeal swabs}

Swab samples were delivered in phosphate buffered saline (PBS) [4]. Samples were directly aliquoted into $1 \mathrm{~mL}$ aliquots, then immediately stored at $-80^{\circ} \mathrm{C}$ and logged (Fig. 4a).

\section{Sputum/saliva}

Samples collected into a collection cup were mixed well at 1:1 ratio with $500 \mathrm{mM}$ DL-Dithiothreitol (DTT) (Sigma)/PBS solution according to CDC recommendations. Diluted samples were then divided into $1 \mathrm{~mL}$ aliquots, volume permitting, immediately stored at $-80^{\circ} \mathrm{C}$ and logged (Fig. 4b).

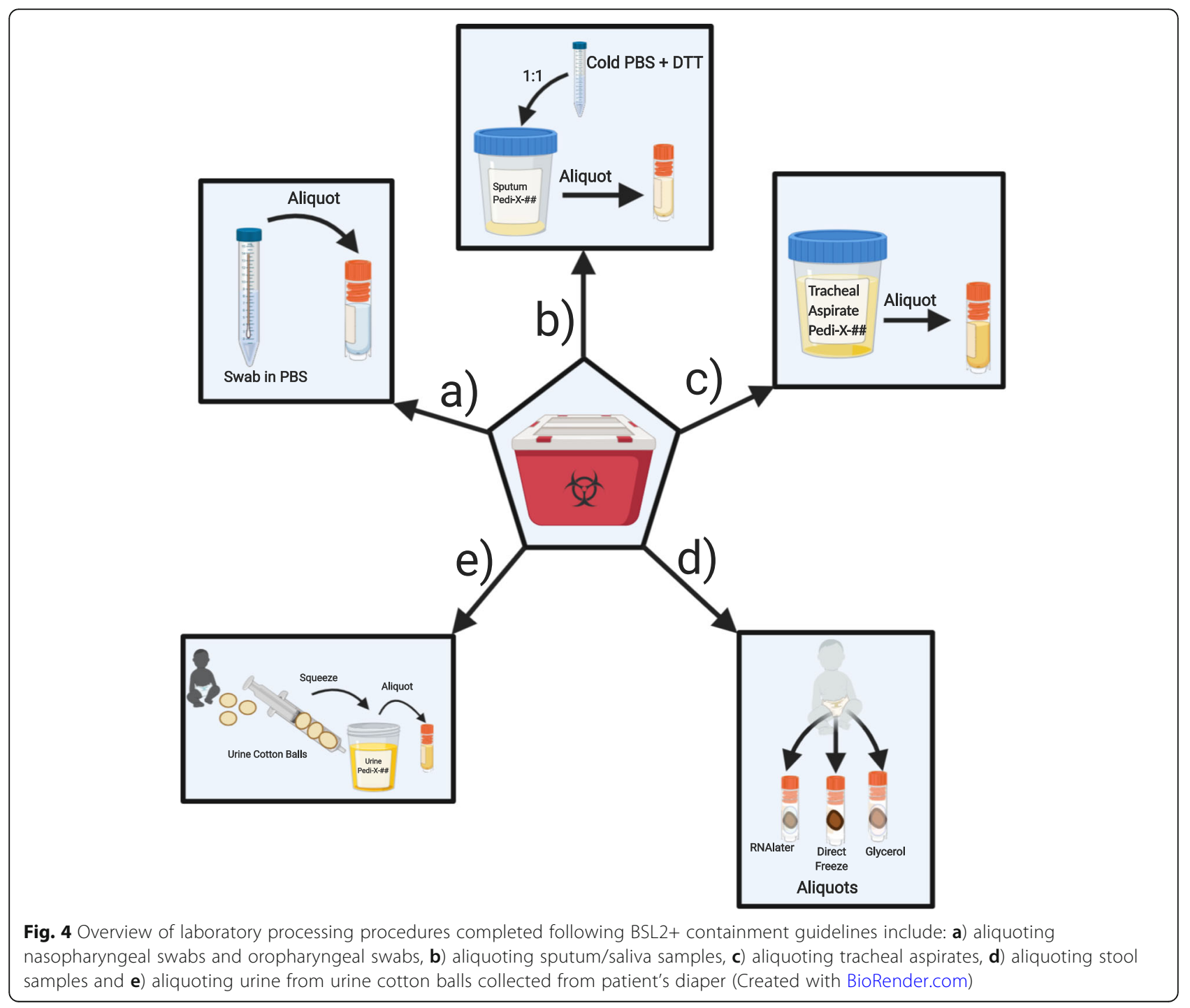




\section{Tracheal aspirates}

Aspirates collected into a sterile collection cup were divided into $1 \mathrm{~mL}$ aliquots $(1 \mathrm{~mL} /$ vial $)$, immediately stored at $-80{ }^{\circ} \mathrm{C}$ and logged (Fig. 4c).

\section{Stool}

Stool samples collected from a diaper or specimen cup were divided using a micro spatula, volume permitting, into cryovials with $1 \mathrm{~mL}$ RNAlater (Invitrogen), empty cryovials without any additive/reagent, up to the $1.5 \mathrm{~mL}$ tube mark, and cryovials with $1 \mathrm{~mL}$ Buffered Glycerol Saline (Fisher). Stool samples were fully submerged in RNAlater or glycerol solution prior to immediate storage at $-80^{\circ} \mathrm{C}$. Samples were logged onto database (Fig. $4 \mathrm{~d}$ ).

\section{Urine}

Urine samples collected with cotton balls placed inside baby diapers were transferred using forceps, to a $10 \mathrm{~mL}$ syringe to dispense at most $1 \mathrm{~mL}$ of fluid into cryovials and immediately stored at $-80^{\circ} \mathrm{C}$. Samples collected into a tube or a sterile collection cup were aliquoted into cryovials (at $1 \mathrm{~mL}$ at most/vial) and immediately stored at $-80^{\circ} \mathrm{C}$ (Fig. 4e).

Supplies required for specimen collection and processing are listed in Supplemental Table 1. Sample labels, logging, storage, and quality control were performed by assigned lab \#3 personnel.

\section{Results}

The Pediatric COVID-19 biorepository enrolled 327 pediatric and neonatal patients from a range of clinical presentations, including 178 patients from the urgent care/Respiratory Infection Control clinic, 48 hospitalized children, 85 newborns born to mothers with or without SARS-CoV-2 infection, and 16 asymptomatic children presenting for their well-visits. The average age was 11 $( \pm 8)$ years for enrolled children and adolescents and 1.3 $( \pm 1.3)$ days for newborns. Equal gender distribution was seen, except more males were enrolled in the hospitalized cohort $(60 \%, n=29)$. Sixty-four participants were positive for SARS-CoV-2 by clinical testing, most of whom were seen in the Respiratory Infection Control clinic, while 21 patients were diagnosed with MIS-C, all of whom were hospitalized. The one patient presenting to the Respiratory Infection Control clinic with MIS-C was ultimately hospitalized. Table 1 characterizes total enrollment number, age, gender, SARS-CoV-2 infection status, and MIS-C diagnosis within each enrollment site.

A total of 972 biospecimens were collected. These biospecimens included 295 blood samples, 181 nasopharyngeal swabs, 145 nasopharyngeal swabs, 172 stool samples, 154 urine samples, 4 tracheal aspirate samples, and $21 \mathrm{sputum} /$ saliva samples. Hospitalized patients and newborns had the option of providing subsequent sampling. Table 2 depicts the sample collection from each enrollment site.

\section{Discussion}

The goal of the biorepository is to provide high quality biospecimens for studies understanding how infants and children are impacted by and contribute to COVID-19 pandemic. Establishing a standardized biorepository collection protocol facilitates comparison of samples across institutions and with adult biorepositories. Key neonatal and childhood factors of interest that will be studied using this biorepository focus on: 1) informing pediatric contribution to viral transmission, 2) teasing apart the dichotomy between pediatric and adult immune responses to COVID-19, 3) ascertaining the impact of maternal SARS-CoV-2 infection on child fetal development, and 4) elucidating factors driving the MIS-C.

\section{Inform pediatric contribution to viral transmission}

In this study, nasopharyngeal and oropharyngeal swabs were collected from pediatric patients presenting with symptoms concerning for SARS-CoV-2 infection in both the outpatient and hospitalized setting, from newborns born to mothers infected with SARS-CoV-2, and from healthy controls. Additionally, saliva was collected from young children presenting for their annual well-visit. Blood, tracheal aspirates, stool, and urine were also collected from the hospitalized patients and newborns for assessment of viral load. Questions relating to the role of viral carriage in the pediatric population can be addressed using these samples. Case reports and recent research studies suggests that asymptomatic children carry high viral loads despite lack of symptoms [5-7]. In adults, severe infection is not necessarily associated with a significant increase in viral loads by nasopharyngeal swab [8], and asymptomatic individuals appear to have equal viral loads as symptomatic individuals [9]. The potential correlations between viral load, symptoms, and exposures have yet to be clarified in the pediatric population. Age-stratification within adults show no differences in viral load across age-groups, although younger patients were less likely to develop severe disease [8], thus a similar comparison among children would be informative. Additional questions remain as to whether there are risk factors affecting viral load density in children, including household contacts or other environmental factors. Viral studies are also needed to determine accuracy of viral testing techniques within the pediatric population. Including infants born to COVID19 infected mothers will allow assessment of viral exposure in the airway, and through the meconium, giving important insight into neonatal exposure to SARS-CoV-2 infection. Understanding how children are infected with SARS-CoV-2 will provide critical insight 
Table 1 Characteristics of enrolled patients

\begin{tabular}{lllll}
\hline $\begin{array}{l}\text { Total enrolled } \\
(\mathbf{N}=\mathbf{3 2 7})\end{array}$ & $\begin{array}{l}\text { Urgent Care } \\
(\boldsymbol{n}=\mathbf{1 7 8})\end{array}$ & $\begin{array}{l}\text { Hospitalized } \\
(\boldsymbol{n}=\mathbf{4 8})\end{array}$ & $\begin{array}{l}\text { Newborn } \\
(\boldsymbol{n}=\mathbf{8 5})\end{array}$ & $\begin{array}{l}\text { Well visit } \\
(\boldsymbol{n}=\mathbf{1 6})\end{array}$ \\
\hline Age, average (SD) & 12.3 years (7.9) & 8.5 years (8.2) & 1.3 days (1.3) & 4.0 years (4.8) \\
\% Male (number) & $50(89)$ & $60(29)$ & $51(43)$ & $50(8)$ \\
SARS-CoV-2 pcr (+) & 46 & 18 & 0 & 0 \\
MIS-C & 1 & 20 & 0 & 0 \\
\hline
\end{tabular}

Age, sex, SARS-CoV-2 clinical testing results, and MIS-C status are described. Polymerase chain reaction of nasopharyngeal swab was clinically used to determine SARS-CoV-2 infection status

into how viral loads may impact disease severity in children, and how children may contribute to viral transmissibility driving this pandemic. These data will be critical to making decisions about risk factors for re-opening of schools and childcare as the pandemic progresses.

Tease apart the dichotomy between pediatric and adult immune responses to COVID-19

COVID-19 results in a major apparent dichotomy of immune response between children and adults [10]. Children often develop mild infections whereas adults more commonly develop severe disease associated with high levels of mortality [11]. Neonates appear to be unaffected, even when born to COVID-19 positive mothers [12]. It has been postulated that children are less impacted by viral infection because they have fewer angiotensin-converting enzyme 2 (ACE2) viral binding sites [7, 13], although the research thus far remains conflicted. In this study, RNA obtained from nasopharyngeal and oropharyngeal swabs, and/or saliva collected from neonates, children, and young adults, can be used to characterize ACE2 expression, and potentially shed light on the availability of viral binding sites across the age span. Further, prior research has shown immunosenescence in aged individuals, which affects $\mathrm{T}$ cell and $\mathrm{B}$ cell function, and cytokine production by innate immune cells [14]. It is yet to be evaluated as to whether this plays a central role in the age differences in the morbidity and mortality from COVID-19. Additionally, MIS-C is shown to be driven by a cytokine storm and macrophage activation [15]. Peripheral blood monocytes, plasma, serum, and neutrophil RNA collected as part of this biorepository can be used to answer these questions. Understanding the disease abnormalities may provide key insight into therapeutic targets. This study collects plasma, serum, PBMCs, and neutrophil RNA from SARS-CoV-2 infected and uninfected children with a range of symptoms for comparison.

\section{Ascertain the impact of maternal SARS-CoV-2 infection on fetal development}

Neonatal development intimately depends on maternal health. Prior infections and disease states causing maternal inflammatory activation and cytokine storm have resulted in increased risk of autism spectrum disorder, schizophrenia, cerebral palsy, cognitive delay, depression, and bipolar disorder in exposed children [16-18]. The effect of the SARS-CoV-2 hyperinflammatory milieu on the developing fetus is yet to be seen. This biorepository, partnered with the Obstetric COVID-19 Biorepository will obtain placental tissue, cord blood, maternal and neonatal biospecimens to address these critical questions.

\section{Elucidate factors driving the multisystem inflammatory syndrome in children (MIS-C)}

Following a mild or symptomatic infection of COVID19, children can develop a severe, post-infectious inflammatory response syndrome, termed Multisystem Inflammatory Syndrome in Children (MIS-C), which is characterized by hyperinflammatory shock [19], "Kawasaki-like" cardiac damage, and possible death [20]. Risk

Table 2 Biospecimens collected from each patient cohort

\begin{tabular}{|c|c|c|c|c|c|c|c|}
\hline $\begin{array}{l}\text { Total number of samples } \\
(\mathbf{N}=\mathbf{9 7 2})\end{array}$ & $\begin{array}{l}\text { Blood } \\
(n=295)\end{array}$ & $\begin{array}{l}\text { Oropharyngeal swab } \\
(\boldsymbol{n}=\mathbf{1 8 1})\end{array}$ & $\begin{array}{l}\text { Nasopharyngeal swab } \\
(\boldsymbol{n}=\mathbf{1 4 5})\end{array}$ & $\begin{array}{l}\text { Stool } \\
(n=172)\end{array}$ & $\begin{array}{l}\text { Urine } \\
(n=154)\end{array}$ & $\begin{array}{l}\text { Tracheal aspirate } \\
(\boldsymbol{n}=\mathbf{4})\end{array}$ & $\begin{array}{l}\text { Sputum/saliva } \\
(\boldsymbol{n}=\mathbf{2 1})\end{array}$ \\
\hline Urgent Care & 86 & 105 & 79 & 0 & 3 & 0 & 7 \\
\hline Hospitalized & 147 & 43 & 44 & 46 & 58 & 3 & 10 \\
\hline Newborn & 55 & 29 & 19 & 126 & 93 & 1 & 0 \\
\hline Well visit & 7 & 4 & 3 & 0 & 0 & 0 & 4 \\
\hline
\end{tabular}

All enrolled participants provided clinical and demographic data. Enrolled subjects had the option of selecting which biospecimens they would like to provide. Stool and urine were not collected from participants enrolled in the Urgent Care and Well-Visit cohorts for logistic reasons, unless these individuals were later hospitalized for COVID-19-related illness. Enrolled subjects could also consent to provide specimens, then later decline any or all specimen collection. Repeat biospecimen collection could occur if participants re-presented to care, or if hospitalized for multiple consecutive days 
factors for developing MIS-C and biomarkers predicting severe complications need to be identified. These specimens collected through this Pediatric COVID-19 biorepository will be used to characterize the immune responses driving MIS-C in hopes of mitigating this lifethreatening complication.

\section{Conclusion}

Although children were initially felt to be spared from COVID-19, it has become clear that much needs to be learned as to how children and newborns are impacted by the pandemic. Research is needed to address viral transmission by children, differences in pediatric viral susceptibility and immune responses, the impact of maternal SARS-CoV-2 infection on fetal development, and factors driving the MIS-C. This Pediatric COVID-19 Biorepository will serve as an important resource providing critical insight into disease pathogenesis, COVID-19susceptibility, and future treatment and vaccination strategies.

\section{Supplementary information}

Supplementary information accompanies this paper at https://doi.org/10. 1186/s12874-020-01110-y.

Additional file 1 Supplemental Table 1. List of supplies required for specimen collection, processing, and storage in the Pediatric COVID-19 Biorepository

\section{Abbreviations \\ ACE2: Angiotensin-converting enzyme 2; ARDS: Acute respiratory distress syndrome; BSC: Biosafety cabinet; BSL (2+): Biosafety level (2 plus); EDTA: Ethylenediamine tetraacetic Acid; FBS: Fetal bovine serum; HBSS: Hank's balanced salt solution; IRB: Institutional review board; IBC: Institutional biosafety committee; L\&D: Labor and delivery; MGH: Massachusetts general hospital; MIS-C: Multisystem Inflammatory syndrome in children; $\mathrm{mL}$ : Milliliters (unit of measurement); PCR: Polymerase chain reaction; PMBC: Peripheral mononuclear blood cells; PBS: Phosphate} buffered solution; PPE: Personal protective equipment; RNA: Ribonucleic acid

\section{Acknowledgements}

We are incredibly grateful for the selfless contributions by the entire Pediatric and Obstetrics COVID-19 Biorepository Team, which includes: Alex Hopke, PhD, Alexandra Kuznetsov, BA, Anika Marand, BS, Andy Yu, BS, Anthony Rosenzweig MD, Brittany Wilson-Mifsud, BS, Bryan Hurley, PhD, Cammie Lesser, MD/PhD, Daniel Irimia, MD/PhD, Doo Yeon Kim, PhD, Dominique Zarrella, BS, Gloria Serena, PhD, Jesiel Lombay, BS, Jessica Tran, BS, Jinggang Lan, PhD, Kristin White, PhD, Linjie Xiong, MD, Marcy Kingsbury, PhD, Maggie Loiselle, BS, Maureen Clark, BS, Nitya Jain, PhD, Natalie Croul, BS, Rosemary Barone, BS, Rudolph Tanzi, PhD, Samantha Devane, BA, Sara Brigida, BA, Tian Lin, MD/PhD, Xingbin Ai, PhD, Yuxin Ma, BS, and the Massachusetts General Hospital Translational and Clinical Research Center.

We are also extremely thankful for the clinicians who recognized the importance of establishing this Biorepository and facilitated the integration of research into the clinical setting: Alexy Arauz Boudreau, MD, Benjamin Nelson, MD, Marjorie Curran, MD, Puneeta Arya, MD, and Rebecca Lambert, MD.

\section{Authors' contributions}

$R L, E G, K H, P L, A F, J S, A E, A N$ and $L M Y$ designed and implemented the biorepository; RL, EG, DD, LY, EB, PD, SN, KH, KG, SG, SD, PF, KH, KH, JZ, GP, $\mathrm{MH}, \mathrm{AN}, J \mathrm{M}, J \mathrm{~S}, \mathrm{AE}, \mathrm{LMY}$ established and adapted the protocols required for the biorepository and led the biorepository collection efforts; RL, EG, LMY wrote the initial draft and finalized the manuscript; All authors edited and approved the final manuscript.

\section{Funding}

We also acknowledge funding from the National Heart Lung and Blood Institute (5K08HL143183 to LY), the Cystic Fibrosis Foundation (YONKER18Q0 to LY), and the Eunice Kennedy Shriver National Institute of Child Health and Human Development (1R01HD100022-01 to AGE). The funder/sponsor did not participate in the work.

Availability of data and materials

A variety of pediatric samples collected during the COVID-19 pandemic may become available to other researchers upon reasonable request to the correspondent author and compliance with the Partners Innovations Office.

Ethics approval and consent to participate

The study received ethics approval from the Partners/Massachusetts General Hospital Institutional Review Board (IRB\#2020P000955) and the Partners/ Massachusetts General Hospital Institutional Biosafety Committee (IBC\#2020B000061). Verbal consent to participate was obtained from the participants or from parents/guardians (for children under 18 years of age). Verbal assent was obtained, when possible, from children ages 7-18 years of age. All participants signed an IRB-approved informed consent prior to participating.

Consent for publication

Not applicable.

\section{Competing interests}

The authors declare no competing interest.

\section{Author details}

${ }^{1}$ Mucosal Immunology and Biology Research Center, Massachusetts General Hospital, Boston, MA, USA. ²Department of Pediatrics, Division of Gastroenterology and Nutrition, Massachusetts General Hospital, Boston, MA, USA. ${ }^{3}$ Department of Pediatrics, Pulmonary Division, Massachusetts General Hospital, Boston, MA, USA. ${ }^{4}$ Department of Internal Medicine, Massachusetts General Hospital, Boston, MA, USA. ${ }^{5}$ Department of Obstetrics and Gynecology, Division of Maternal-Fetal Medicine, Vincent Center for Reproductive Biology, Massachusetts General Hospital, Boston, MA, USA. ${ }^{6}$ Department of Pediatrics, Lurie Center for Autism, Massachusetts General Hospital, Boston, MA, USA. Department of Neurology, Massachusetts General Hospital, Boston, MA, USA. ${ }^{8}$ Department of Dermatology, Cutaneous Biology Research Center, Massachusetts General Hospital, Boston, MA, USA.

Translational and Clinical Research Center, Massachusetts General Hospital, Boston, MA, USA. ${ }^{10}$ Department of Pediatrics, Infectious Disease, Massachusetts General Hospital, Boston, MA, USA. ${ }^{11}$ Department of Pediatrics, Division of Neonatology and Newborn Medicine, Massachusetts General Hospital, Boston, MA, USA. ${ }^{12}$ Harvard Medical School, Boston, MA, USA.

Received: 13 July 2020 Accepted: 30 August 2020

Published online: 11 September 2020

\section{References}

1. World Health Organization. https://www.who.int/emergencies/diseases/ novel-coronavirus-2019. Accessed 4 Sept 2020

2. Commonwealth of Massachusetts COVID-19 reporting. https://www.mass. gov/info-details/covid-19-response-reporting. Accessed 4 Sept 2020.

3. Boyum A. Isolation of lymphocytes, granulocytes and macrophages. Scand J Immunol. 1976;5(Suppl 5):9-15

4. Druce J, Garcia K, Tran T, Papadakis G, Birch C. Evaluation of swabs, transport media, and specimen transport conditions for optimal detection of viruses by PCR. J Clin Microbiol. 2012;50(3):1064-5.

5. Kam KQ, Yung CF, Cui L, Lin Tzer Pin R, Mak TM, Maiwald M, Li J, Chong CY, Nadua K, NWH T, et al. A well infant with coronavirus disease 2019 (COVID19) with high viral load. Clin Infect Dis. 2020;71(15):847-9.

6. Han MS, Seong MW, Kim N, Shin S, Cho SI, Park H, Kim TS, Park SS, Choi EH. Viral RNA load in mildly symptomatic and asymptomatic children with COVID-19, Seoul. Emerg Infect Dis. 2020;26(10). Online, ahead of print. 
7. Yonker LM, Neilan AM, Bartsch Y, Patel AB, Regan J, Arya P, Gootkind E, Park G, Hardcastle M, St John A, et al. Pediatric SARS-CoV-2: clinical presentation, infectivity, and immune responses. J Pediatr. 2020;S0022-3476(20):31023-4.

8. Shi F, Wu T, Zhu X, Ge Y, Zeng X, Chi Y, Du X, Zhu L, Zhu F, Zhu B, et al. Association of viral load with serum biomakers among COVID-19 cases. Virology. 2020;546:122-6.

9. Zou L, Ruan F, Huang M, Liang L, Huang H, Hong Z, Yu J, Kang M, Song Y, Xia J, et al. SARS-CoV-2 viral load in upper respiratory specimens of infected patients. N Engl J Med. 2020;382(12):1177-9.

10. Fialkowski A, Gernez Y, Arya P, Weinacht KG, Kinane TB, Yonker LM. Insight into the pediatric and adult dichotomy of COVID-19: age-related differences in the immune response to SARS-CoV-2 infection. Pediatr Pulmonol. 2020. https://doi.org/10.1002/ppul.24981.

11. Team CC-R. Coronavirus disease 2019 in children - United States, February 12-April 2, 2020. MMWR Morb Mortal Wkly Rep. 2020:69(14):422-6.

12. Thomas $\mathrm{P}$, Alexander $\mathrm{PE}$, Ahmed $\mathrm{U}$, Elderhorst $\mathrm{E}$, El-Khechen $\mathrm{H}$, Mammen MJ, Debono VB, Aponte Torres Z, Aryal K, Brocard E, et al. Vertical transmission risk of SARS-CoV-2 infection in the third trimester: a systematic scoping review. J Matern Fetal Neonatal Med. 2020:1-8.

13. Bunyavanich S, Do A, Vicencio A. Nasal gene expression of angiotensinconverting enzyme 2 in children and adults. JAMA. 2020;323(23):2427-9.

14. Aw D, Silva $A B$, Palmer DB. Immunosenescence: emerging challenges for an ageing population. Immunology. 2007;120(4):435-46

15. Cheung EW, Zachariah P, Gorelik M, Boneparth A, Kernie SG, Orange JS, Milner JD. Multisystem inflammatory syndrome related to COVID-19 in previously healthy children and adolescents in New York City. JAMA. 2020.

16. Al-Haddad BJS, Jacobsson B, Chabra S, Modzelewska D, Olson EM, Bernier R, Enquobahrie DA, Hagberg H, Ostling S, Rajagopal L, et al. Long-term risk of neuropsychiatric disease after exposure to infection in utero. JAMA Psychiatry. 2019;76(6):594-602.

17. Al-Haddad BJS, Oler E, Armistead B, Elsayed NA, Weinberger DR, Bernier R, Burd I, Kapur R, Jacobsson B, Wang C, et al. The fetal origins of mental illness. Am J Obstet Gynecol. 2019;221(6):549-62.

18. Kepinska AP, lyegbe CO, Vernon AC, Yolken R, Murray RM, Pollak TA. Schizophrenia and influenza at the centenary of the 1918-1919 Spanish influenza pandemic: mechanisms of psychosis risk. Front Psychiatry. 2020;11:72.

19. Riphagen S, Gomez X, Gonzalez-Martinez C, Wilkinson N, Theocharis P. Hyperinflammatory shock in children during COVID-19 pandemic. Lancet. 2020;395(10237):1607-8.

20. Feldstein LR, Rose EB, Horwitz SM, Collins JP, Newhams MM, Son MBF, Newburger JW, Kleinman LC, Heidemann SM, Martin AA, et al. Multisystem inflammatory syndrome in U.S. children and adolescents. N Engl J Med. 2020;383(4):334-46.

\section{Publisher's Note}

Springer Nature remains neutral with regard to jurisdictional claims in published maps and institutional affiliations.

Ready to submit your research? Choose BMC and benefit from:

- fast, convenient online submission

- thorough peer review by experienced researchers in your field

- rapid publication on acceptance

- support for research data, including large and complex data types

- gold Open Access which fosters wider collaboration and increased citations

- maximum visibility for your research: over $100 \mathrm{M}$ website views per year

At $\mathrm{BMC}$, research is always in progress.

Learn more biomedcentral.com/submissions 